\title{
Three-dimensional phase-field study of grain coarsening and grain shape accommodation in the final stage of liquid-phase sintering
}

\author{
Hamed Ravash $^{\mathrm{a}}$, Liesbeth Vanherpe ${ }^{\mathrm{b}}$, Jef Vleugels ${ }^{\mathrm{a}}$, Nele Moelans ${ }^{\mathrm{a}, *}$ \\ ${ }^{a}$ KU Leuven, Department of Materials Engineering, Kasteelpark Arenberg 44, bus2450, \\ 3001 Leuven, Belgium \\ ${ }^{b}$ Blue Brain Project, EPFL, Chemin des Mines 9, CH-1211 Geneva, Switzerland
}

\begin{abstract}
A 3-dimensional phase-field model is implemented to simulate the grain evolution in the final stage of liquid-phase sintering. The model considers a liquid phase and a polycrystalline solid phase. Results for varying ratios of the solid-solid interface energy to solid-liquid interface energy and varying solid volume fractions are presented. A variety of microstructures, from fully connected grain structures with liquid pockets at the grain junctions to individual grains fully wetted by the liquid matrix, is seen. The 3 main mechanisms for particle shape accommodation, namely, contact flattening, Ostwald ripening and particle bonding, are reproduced in the simulations. The solid volume fraction, particle size distribution, contiguity, connectivity, particle-particle contact areas and the number of particle contacts per particle are measured as a function of time. The exponent in the power growth law varies between 2.4, for the fully connected grain structures, and 3, for the completely wetted grains.
\end{abstract}

Keywords: 3-d microstructure simulation, liquid phase sintering, Ostwald ripening, grain growth wetting, annealing, phase-field model, multi-phase system

\footnotetext{
${ }^{*}$ Corresponding author

Email address: nele.moelans@kuleuven. be (Nele Moelans)
} 


\section{Introduction}

For materials which are difficult to sinter with the classical approach, liquid phase sintering (LPS), also called liquid phase assisted sintering, is commonly used. In liquid phase sintering, additives are added to the powder to form a liquid phase at temperatures considerably lower than the melting point of the matrix phase. The method is now also widely employed for fabrication of $3 \mathrm{D}$ parts in the powder based additive manufacturing [1]. In the final stage of liquid-phase sintering, microstructural evolution of the system is controlled by Ostwald ripening and grain growth.

For a virtually zero particle fraction, the coarsening rate of the particles has been predicted using the analytical models of Greenwood [4], Lifshitz and Slyozov [5], and Wagner [6] in the so-called LSW theory. Later, Ardell [7] and Tsumuraya [8] developed a LSW theory for finite solid volume fractions and others $[9,10,11,12]$ incorporated assumptions on the grain shape and the diffusion field around the particles. Very recently, coarsening in systems with high and ultra-high volume fractions (up to 0.96) have been studied using experimental and three-dimensional simulation methods $[13,14,15,16,17,18]$. While there exist extensive 3-d simulation studies of microstructure evolution during solid-state sintering [19, 20, 21, 22, 23], the studies for liquid-phase sintering are still limited $[24,25,26]$. In general, a particle volume fraction larger than 60 vol.\% is required for an appropriate geometrical control in liquid phase sintering $[2,3]$, which signifies the importance of a further study of the coarsening processes in solid-liquid systems with high solid volume fractions .

The volume fraction of the solid phase, together with the relative grain boundary and solid-liquid interface energies have a clear effect on the grain shapes and connectivity of the grain structure [3]. For example, in isotropic systems with particle volume fraction $f_{p}<70$ vol.\%, the grains have an almost spherical shape, except at their contacts with other particles [3]. For higher fractions of the solid phase, the grain faces flatten to eliminate pores

and the liquid forms pockets at the grain boundary junctions. The shape of the liquid pockets is determined by the ratio of the grain boundary energy of the solid phase, $\sigma_{s s}$, and the solid-liquid interface energy, $\sigma_{s l}$. Furthermore, the contacts between two particles grow until they have reached an equilibrium size, called the stable grain-grain contact size, which depends on the particle sizes and the ratio of the solid-liquid and solid-solid interface energies [3]. 
The mean apparent dihedral angle $\phi$ at a particle-particle contact $[3,27$, $28,29]$ is defined as illustrated in Fig. 1 and given by $2 \sigma_{s l} \cos (\phi / 2)(1-s / r)=$ $\sigma_{s s}$, with $r$ the neck radius and $s$ neck surface radius of curvature. During the neck growth at particle-particle contacts, the apparent or measured dihedral angle may be different from the theoretically expected dihedral angle $\phi^{*}$, as obtained from Young's law $\cos \left(\phi^{*} / 2\right)=0.5 \sigma_{s s} / \sigma_{s l}$. The apparent and equilibrium dihedral angles relate as $\cos (\phi / 2)=\cos \left(\phi^{*} / 2\right)+t / X$, with $t$ the neck thickness and $X=2 r$. In general, a low mean apparent dihedral angle promotes wetting of the grains and facilitates densification. Correlations have been found between the mean apparent dihedral angle and contiguity and connectivity $[31,32]$. The contiguity $C_{s s}$ is defined as the fraction of solidsolid interface contact area in the microstructure with respect to the total amount of solid-solid and solid-liquid interface area [30]. The connectivity is defined as the average number of particle-particle connections per particle as observed on a random $2 \mathrm{D}$ section.

In the presented work, we have performed 3-dimensional simulations based on an adapted phase-field model considering particle volume fractions ranging between $f_{p}=0.65$ and $f_{p}=0.83$, and solid-solid to solid-liquid interface energy ratios $\sigma_{s s} / \sigma_{s l}$ ranging between 1 and 2.5. The important microstructural characteristics were determined as a function of time and particle volume fraction and solid-solid to liquid-solid interface energy ratio. The simulation results are compared with existing empirical laws and different sets of experimental data.

\section{Model description}

A novel phase-field model is presented, specifically designed to model the liquid phase assisted grain coarsening and grain shape accommodation phenomena in the final stage of LPS. Essentially, the model is an assembly of the interfacial energy description of Moelans et al. $[33,34]$ with a bulk energy description based on the formulation of Folch and Plapp [35].

The interfacial energy description of Moelans et al. allows for an accurate description of interfacial and grain boundary energies in polycrystalline multi-phase structures with inhomogeneous interface properties and avoids the presence of so-called 'third' or 'ghost' phases [36, 37] at grain boundaries or interfaces affecting their properties and kinetics.

A great advantage of the bulk energy formulation of Folch and Plapp is that it does not affect the interface properties in the model. It also avoids 
the presence of solute segregation at grain boundaries of which the magnitude is difficult to control in phase-field models when the diffuse interface width is taken larger than the physical interface width, which is often required because of computational limitations. The onrealistically large solute segregation could affect the kinetics of interface and grain boundary movement [38, 39]. The bulk energy formulation of Folch and Plapp assumes a parabolic composition dependence. Although, strictly speaking, a parabolic composition dependence is thermodynamically inconsistent in the dilute limits $c_{i} \rightarrow 0$ and $c_{i} \rightarrow 1$ and generally does not describe the composition dependence well over a large composition range, it is reasonable to apply this simplification when considering coarsening phenomena in bulk materials, since in these processes the compositions of the phases remain close to the equilibrium compositions.

As in the models of $[12,33,40]$, the presented model uses a large set of non-conserved phase-field variables, $\eta_{1}(\mathbf{r}, t), \ldots, \eta_{p_{s}}(\mathbf{r}, t)$, which are a function of space $\mathbf{r}$ and time $t$, representing the different grain orientations in the solid phase. $p_{s}$ is taken equal to the number of solid phase particles considered in the simulations. An extra non-conserved phase-field variable, denoted as $\eta_{p_{s}+1}(\mathbf{r}, t)$, is introduced to represent the liquid matrix phase. The nonconserved phase-field variables are defined such that in each grain, one of the $\eta_{i}$ (with $i=1, \ldots, p_{s}$ ) equals 1 , namely that representing the considered grain, while all the other phase-field variables equal 0. Similarly, within the liquid phase, $\eta_{p_{s}+1}=1$ and $\eta_{i \neq p_{s}+1}=0$. At grain boundaries and solid-liquid interfaces, the phase-field variables change their values smoothly. The region where this happens is called the diffuse interface region. Its width is typically chosen based on computational considerations. These phase-field variables are not interpreted as phase fractions and their sum can deviate from 1 over the diffuse interface regions. They are merely used to indicate which phase(s) is(are) present at each point.

The composition variations in the system are described using one conserved phase-field variable $c(\mathbf{r}, t)$, representing the local molar fraction of one of the 2 components (further referred to as the independent component; the other component is called the dependent component) as a function of space and time.

The thermodynamic free energy of the system is modeled to consist of bulk $\left(F_{\text {chem }}\right)$ and interface $\left(F_{\text {inter }}\right)$ contributions, namely

$$
F=F_{\text {chem }}+F_{\text {inter }} .
$$


Pressure $P$, temperature $T$ and molar volume $V_{m}$ are constant throughout the system and in time.

The chemical bulk energy of the system is formulated following the approach of Folch and Plapp [35], giving

$$
F_{\text {chem }}=\int_{V}\left[f_{\text {chem }}\left(\eta_{1}, \ldots, \eta_{p_{s}+1}, c\right)\right] d V
$$

where the integral is taken over the simulation domain with volume $V$ and with $f_{\text {chem }}$ as a function of the phase fields $\eta_{1, \ldots, p_{s}+1}(\mathbf{r}, t)$ and concentration field $c(\mathbf{r}, t)$,

$$
\begin{aligned}
f_{\text {chem }}\left(\eta_{1}, \ldots, \eta_{p_{s}+1}, c\right) & =\frac{A}{2}\left(c-\left(c_{s}^{0} H_{s}\left(\eta_{1}, \ldots, \eta_{p_{s}+1}\right)+c_{l}^{0} H_{l}\left(\eta_{1}, \ldots, \eta_{p_{s}+1}\right)\right)\right)^{2} \\
& +\left(G_{s} H_{s}\left(\eta_{1}, \ldots, \eta_{p_{s}+1}\right)+G_{l} H_{l}\left(\eta_{1}, \ldots, \eta_{p_{s}+1}\right)\right)
\end{aligned}
$$

where $c_{s}^{0}, c_{l}^{0}, G_{s}, G_{l}$ and $A$ are model parameters chosen to reproduce the equilibrium compositions of the solid and liquid phase. $H_{s, l}\left(\eta_{1}, \ldots, \eta_{p_{s}+1}\right)$ are functions that interpolate between the composition dependent energy descriptions of the solid and the liquid. As specified by Folch and Plapp, these functions should be chosen such that they obey the following requirements: $H_{l}=1$ in the liquid phase (i.e. $\left.\eta_{p_{s}+1}=1, \eta_{i \neq p_{s}+1}=0\right), \mathrm{H}_{l}=0$ in the solid phase and $\partial H_{l} / \partial \eta_{i}=0, i=1 \ldots p_{s}+1$ in both the solid and liquid phase (i.e. where one of the $\eta_{i}$ equals 1 and the other 0 ) and, similarly, $H_{s}=1$ in the solid phase, $H_{s}=0$ in the liquid phase and $\partial H_{s} / \partial \eta_{i}=0, i=1 \ldots p_{s}+1$ in both the solid and the liquid. As the interpolation functions proposed by Folch and Plapp do not extend easily to polycrystalline structures [35], the interpolation functions of Moelans [41]

$$
\begin{aligned}
& H_{s}\left(\eta_{1}, \ldots, \eta_{p_{s}+1}\right)=\varphi_{s}\left(\eta_{1}, \ldots, \eta_{p_{s}+1}\right)=\frac{\sum_{j=1}^{p_{s}} \eta_{j}^{2}}{\left(\sum_{j=1}^{p_{s}} \eta_{j}^{2}+\eta_{p_{s}+1}{ }^{2}\right)} \\
& H_{l}\left(\eta_{1}, \ldots, \eta_{p_{s}+1}\right)=\varphi_{l}\left(\eta_{1}, \ldots, \eta_{p_{s}+1}\right)=\frac{\eta_{p_{s}+1}^{2}}{\left(\sum_{j=1}^{p_{s}} \eta_{j}^{2}+\eta_{p_{s}+1}{ }^{2}\right)}
\end{aligned}
$$

were used, which obey all requirements specified by Folch and Plapp. Furthermore, it follows from their definition that the interpolation functions $H_{s}$ 
and $H_{l}$ can be interpreted as the local phase fractions $\varphi_{s}$ and $\varphi_{l}$ of the solid and liquid phase, respectively.

The interfacial energy contribution is taken from Moelans [41]. This energy formulation is based on the grain growth models originally proposed by Chen and Yang [42] and Kazaryan et al.[40]. The further modifications to this model and relations between model parameters and grain boundary or interface properties proposed by Moelans et al. [33], Tang et al. [43] and Chang and Moelans [44] are also applied in this work. The interfacial energy is then included as

$$
F_{\text {interf }}=\int_{V}\left[f_{\text {interf }}\left(\eta_{1}, \ldots, \eta_{p_{s}+1}\right)\right] d V
$$

with

$$
f_{\text {inter }}=m\left[\sum_{i=1}^{p_{s}+1}\left(\frac{\eta_{i}^{4}}{4}-\frac{\eta_{i}^{2}}{2}\right)+\sum_{i=1}^{p_{s}+1} \sum_{j=i+1}^{p_{s}+1} \gamma_{i, j} \eta_{i}^{2} \eta_{j}^{2}+\frac{1}{4}\right]+\frac{\kappa}{2} \sum_{i=1}^{p_{s}+1}\left(\nabla \eta_{i}\right)^{2}
$$

where the summations are taken over all phase-field variables representing the solid and the liquid phase. The model parameters $m, \gamma_{i, j}$ and $\kappa$ are related to the interfacial energies $\sigma_{i, j}$ and diffuse interface widths $l_{i, j}$ of the boundaries between the different grains or phases. As described in [33], for a grain boundary between two grains represented by $\eta_{i}$ and $\eta_{j}$, the grain interface energy can be written as $\sigma_{i, j}=g\left(\gamma=\gamma_{i, j}\right) \sqrt{\kappa m}$ and the diffuse interface width as $l_{i, j}=\sqrt{\kappa /\left(m f_{0, \max }\left(\gamma=\gamma_{i, j}\right)\right)}$. This $g(\gamma)$ has been evaluated numerically for a wide range of $\gamma$-values. The width of the diffuse interface region $l_{i, j}$ is defined as the reverse of the maximum gradient to the $\eta_{i}$ and $\eta_{j}$ profiles perpendicular to their interface (reached at the middle of the interface region). The values of $f_{0, \max }(\gamma)$ were determined from the equilibrated 1-dimensional two-grain systems as the maximum of the energy profile at the middle of the diffuse interface region. A list of the numerically evaluated function values is available as supplementary material and can be downloaded from [45]. In the presented model, only two types of interfaces with a different energy are present, namely the grain boundaries between solid-phase particles and solid-liquid interfaces.

The evolution of the conserved composition field $c$ is given by the following partial differential equation 


$$
\begin{aligned}
\frac{1}{V_{m}} \frac{\partial c(\mathbf{r}, t)}{\partial t}= & \nabla \cdot\left[\left[H_{s}\left(\eta_{1}, \ldots, \eta_{p_{s}+1}\right) M_{s}(c)+H_{l}\left(\eta_{1}, \ldots, \eta_{p_{s}+1}\right) M_{l}(c)\right]\right. \\
& \left.\nabla\left[\widetilde{\mu}\left(\eta_{1}, \ldots, \eta_{p_{s}+1}, c\right)\right]\right]
\end{aligned}
$$

with $\widetilde{\mu}$ the diffusion potential given as $V_{m} \partial f_{c h e m} / \partial c, M_{s}$ and $M_{l}$ are the diffusion mobilities for respectively the solid and liquid phase and $V_{m}$ is the constant value assumed for the molar volume. Using the formalism of Andersson and Agren [46] for diffusion in multi-component systems, the diffusion mobilities are expressed as a function of atomic mobilities $\beta_{s / l}$ and $\beta_{s / l}^{d}$ of the independent and dependent component in the solid and liquid phase :

$$
M_{s / l}=\left(c(1-c)^{2} \beta_{s / l}+c^{2}(1-c) \beta_{s / l}^{d}\right) 1 / V_{m} .
$$

The evolution equation for the non-conserved phase-fields $\eta_{i, s}$ and $\eta_{l}$ follows a Ginzburg-Landau type of equation,

$$
\frac{\partial \eta_{i}}{\partial t}=-L \frac{\delta F}{\delta \eta_{i}}
$$

where $F$ is the total free energy functional (Eqns. (3) and (6)) and

$$
L=\left[\sum_{i=1}^{p_{s}+1} \sum_{j=i+1}^{p_{s}+1} L_{i, j} \eta_{i}^{2} \eta_{j}^{2}\right] /\left[\sum_{i=1}^{p_{s}+1} \sum_{j=i+1}^{p_{s}+1} \eta_{i}^{2} \eta_{j}^{2}\right]
$$

is a function of kinetic constants related to the grain boundary and interface mobilities. For the solid-solid interfaces, $L_{i, j}$ is related to the grain boundary mobility $\mu_{i, j}$ as $L_{i, j} \kappa=\sigma_{i, j} \mu_{i, j}$ [33]. For the solid-liquid interfaces, $L_{i, j}$ should be chosen so that the velocity of the interface in the phase-field model is controlled by the diffusion in the bulk phases, assuming bulk diffusion controlled conditions. This is the case when [47]

$$
L_{i, j}=L_{i, j}^{\mathrm{eq}}=\frac{\sqrt{2} m g\left(\gamma_{i, j}\right)}{\kappa I\left(\gamma_{i, j}\right) \zeta}
$$

with $\zeta=\left(2 /\left(M_{s}+M_{l}\right)\right)\left(c_{s}^{\text {eq }}-c_{l}^{\text {eq }}\right)^{2}$ for a binary two-phase solid-liquid system and $I\left(\gamma_{i, j}\right)$ and $g\left(\gamma_{i, j}\right)$ functions which have been evaluated numerically and are listed in the supplementary material and at [45]. Inserting Eqs. (3) 
and (6) in Eq. (9) and taking the partial derivative of the energy functional with respect to the considered non-conserved phase-field variable gives the following equation for each phase-field variable $\eta_{i}$

$$
\begin{aligned}
\frac{\partial \eta_{i}}{\partial t}=-L\{m & \left(\eta_{i}^{3}-\eta_{i}+2 \gamma \sum_{j=1, j \neq i}^{p_{s}+1} \eta_{i} \eta_{j}^{2}\right)-\kappa \nabla^{2} \eta_{i} \\
+A & {\left[\left(c-\left(c_{s}^{0} H_{s}+c_{l}^{0} H_{l}\right)\right)\left(-c_{s}^{0} \frac{\partial H_{s}}{\partial \eta_{i}}-c_{l}^{0} \frac{\partial H_{l}}{\partial \eta_{i}}\right)\right] } \\
+ & \left.\left(G_{s}(T) \frac{\partial H_{s}}{\partial \eta_{i}}+G_{l}(T) \frac{\partial H_{l}}{\partial \eta_{i}}\right)\right\} .
\end{aligned}
$$

\subsection{Implementation details}

The bounding box algorithm based on a sparse data structure representation developed by Vanherpe et al. [48, 49] was extended for two-phase systems. For the bounding box algorithm, the computational requirements only scale with the system size and not with the number of phase-field variables used to represent the microstructure, as is the case for conventional algorithms based on the full representation of the data. For the presented simulations, an explicit finite difference method with Forward-Euler time stepping and a second-order central scheme for the Laplacian was used to discretize the partial differential equations (12) and (7).

To generate an initial microstructure with randomly distributed densely packed particles without overlap, a continuum description of the initial particle packing was first generated using a threshold algorithm with iterative improvement $[50,51,52]$, considering particles with radii of 3,6 and 12 such that there are in total 5000 particles and a target solid volume fraction. Then this description is discretized by rounding the coordinates of the centers of the spheres and their radii to the nearest integers. If overlap between two particles is introduced in this discretization step, the radius of one of the particles involved is reduced with one grid unit. Then the particle volume fraction of the discretized structure is calculated again to obtain the initial particle volume fraction in the system.

For the characterization of the simulated microstructures, a discrete voxelbased representation was generated from the resulting phase-fields $\eta_{i}(\mathbf{r})$ at 
different time steps, by taking at each grid point the number of the phasefield with the largest value. From this discrete voxel-based representation, various microstructure measures were determined. The particle volumes were measured by counting the number of voxels belonging to each particle in the discrete voxel-based representation. The equivalent particle radius was then calculated as the radius of a sphere with the same volume as the particle. The areas of solid-liquid and solid-solid interfaces is obtained from the discrete voxel-based representation by counting the number of voxels located next to a solid-liquid or solid-solid interface (and divide this number by 2 since the voxels at the 2 sides of an interfaces are counted ). The contact diameter was calculated as the diameter of a circle with an area equal to the measured area of particle-particle contacts. The number of contacting particles of a particle can be determined by counting the number of unique representation numbers over the neighboring voxels of the grain.

Fig. 2(a) presents the initial structure used for a simulation with $f_{p}=$ 0.78. Fig. 2(b) visualizes the 3 -D discrete representation of the simulated microstructure obtained for $\sigma_{s s}=2 \sigma_{s l}$ and $f_{p}=0.7$ at $t=50 \times 10^{3} \triangle t$.

\subsection{Input parameters}

A model binary 2-phase system is considered in the simulations. To obtain particle volume fractions $f_{p}=0.65,0.70,0.78$ and 0.83 in the coarsening microstructure, the initial average particle sizes and initial supersaturated liquid composition were, respectively, chosen as $\left\langle r_{p}\right\rangle=5.94 \triangle x$ and $c_{l}^{0}=$ $0.85,\left\langle r_{p}\right\rangle=6.31 \triangle x$ and $c_{l}^{0}=0.85,\left\langle r_{p}\right\rangle=6.71 \triangle x$ and $c_{l}^{0}=0.85$, and $\left\langle r_{p}\right\rangle=6.71 \triangle x$ and $c_{l}^{0}=0.88$. In real systems, the liquid is typically not supersaturated in the final stage of LPS. However, for the simulations, it was necessary to start from a supersaturated liquid to be able to reach the desired solid volume fractions, which are quite high. As will be shown in the results sections, the excess solute precipitates very fast at the beginning of the simulations. The parametes in the bulk chemical energy were taken as $c_{s}^{0}=0.95, c_{l}^{0}=0.8, G_{s}=-4 \times 10^{4} \mathrm{~J} / \mathrm{m}^{3}, G_{l}=-8 \times 10^{4} \mathrm{~J} / \mathrm{m}^{3}$ and $A=5 \times 10^{8} \mathrm{~J} / \mathrm{m}^{3}$. Application of the common tangent construction to these energy functions gives $c_{s}^{e q}=0.95005$ and $c_{l}^{e q}=0.8005$ for the equilibrium compositions of the solid and liquid phase. The atomic diffusion mobilities of the independent and dependent elements in the solid and liquid phase are taken as $\beta_{s} / V_{m}=1 \times 10^{-11} \mathrm{~mol}^{2} / \mathrm{mJs}, \beta_{s}^{d} / V_{m}=2 \times 10^{-11} \mathrm{~mol}^{2} / \mathrm{mJs}, \beta_{l} / V_{m}=$ $1 \times 10^{-9} \mathrm{~mol}^{2} / \mathrm{mJs}$ and $\beta_{l}^{d} / V_{m}=2 \times 10^{-9} \mathrm{~mol}^{2} / \mathrm{mJs}$. The grain boundary energy between the solid particles is $\sigma_{s s}=0.25 \mathrm{~J} / \mathrm{m}^{2}$ in all simulations, and 
different values of the solid-liquid interface energy are considered, namely $\sigma_{s l}=0.25 \mathrm{~J} / \mathrm{m}^{2}, \sigma_{s l}=0.147 \mathrm{~J} / \mathrm{m}^{2}, \sigma_{s l}=0.125 \mathrm{~J} / \mathrm{m}^{2}$ and $\sigma_{s l}=0.1 \mathrm{~J} / \mathrm{m}^{2}$ for simulations with different solid-solid to solid-liquid interface energy ratios. In all simulations, the gradient energy coefficient is $\kappa=1.87 \times 10^{-8}$. For the solid-solid interface, $\gamma_{i, j}=\gamma_{s, s}=1.4974$ and $L_{i, j}=L_{s, s}=1 \times 10^{-4}$ in all simulations. For simulations with $\sigma_{s s} / \sigma_{s l}=1,1.7,2$ and 2.5, the model parameters $\gamma_{i, j}=\gamma_{s, l}, L_{i, j}=L_{s, l}$ (for the solid-liquid interface) and $m$ are taken $\gamma_{s, l}=1.4974, L_{s, l}=0.0191$ and $m=0.9 \times 10^{7}\left(\right.$ for $\left.\sigma_{s s} / \sigma_{s l}=1\right)$; $\gamma_{s, l}=0.6977, L_{s, l}=0.0104$ and $m=1.8 \times 10^{7}\left(\right.$ for $\left.\sigma_{s s} / \sigma_{s l}=1.7\right) ; \gamma_{s, l}=$ $0.6332, L_{s, l}=0.0159$ and $m=3.6 \times 10^{7}\left(\right.$ for $\left.\sigma_{s s} / \sigma_{s l}=2\right)$; and $\gamma_{s, l}=0.5796$, $L_{s, l}=0.0109$ and $m=3.6 \times 10^{7}$ (for $\sigma_{s s} / \sigma_{s l}=2.5$ ). A threshold value for the bounding box of $\epsilon=10^{-5}$ was used. The system size was taken $256 \times 256 \times 256$ grid points with grid size $\triangle x=6 \times 10^{-8} \mathrm{~m}$. A time step $\triangle t=1.8 \times 10^{-6}$ s was used.

The choice of the parameters $l_{i, j}$ is a tradeoff between having a good resolution of the diffuse interface profiles, requiring sufficiently large $l_{i, j}$, and reducing diffuse interface effects from overlapping fields in small grains or regions, requiring sufficiently small $l_{i, j}$. As shown in [41], Ostwald ripening is accurately described in the simulations for $l_{i, j} \geq 3 \Delta x$ and $l_{i, j} \leq R$, with $R$ the radius of the smallest particle. Furthermore, it was shown in [33] for grain boundary migration that diffuse interface effects are negligible for $R>2.6 l_{i, j}$, with $R$ the radius of grain boundary curvature, and that there is an error of approximately $5 \%$ on the grain boundary velocity for $l_{i, j} \approx 3 \Delta x$. For the model parameters given above, the diffuse boundary width $l_{i, j}$ is approximately $3 \Delta x$ and approximately 5 times smaller than the average grain radius of the particles from time $t=10^{5} \Delta t$. Since we are considering connected grain structures, there are always (disappearing) grains present in the simulations with a size below the threshold for which diffuse interface effects exist. These grains typically shrink at a rate too fast in the simulations. However, it was shown in [36] that the effect of the fast shrinkage of small grains is only visible in the immediate environment of the grains and is temporarily. Hence, growth rates and grain size distributions obtained from phase-field simulations are generally found to agree well with those theoretically expected. 


\section{Results}

\subsection{Microstructure evolution}

In a first series of simulations, four solid particles surrounding a liquid region were considered using the input parameters given in the previous section. Fig. 3 presents a series of images obtained from simulations for different $\sigma_{s s} / \sigma_{s l}$ ratios. The first row of pictures visualizes the composition map, whereas the second row presents the grain structure using $\sum_{i=1}^{p_{s}+1} \eta_{i}^{2}$, where the summation is taken over all phase-fields considered in the simulation. The series of images clearly reveals that grain wetting is enhanced for increasing $\sigma_{s s} / \sigma_{s l}$ ratio. In agreement with Youngs law, wetting starts from $\sigma_{s s} / \sigma_{s l}=2$, as shown in Fig. 3(d) and Fig. 3(i). Clear wetting is seen in the simulation for $\sigma_{s s} / \sigma_{s l}=2.5$ (Figs. $3(\mathrm{e})$ and $(\mathrm{j})$ ). While a high $\sigma_{s s} / \sigma_{s l}$ results in a liquid pocket with concave interfaces, as shown in Fig. 3(e), the liquid pocket has convex interfaces at low $\sigma_{s s} / \sigma_{s l}$ values, as presented in Fig. 3(a).

Next, large-scale simulations were performed starting from a system with initially approximately 5000 spherical solid particles $\left(p_{s}=5000\right)$ randomly distributed in a supersaturated liquid matrix without overlap. The simulated systems were followed over 250000 time steps. Every 2000 time steps, the particle size distribution, solid volume fraction, contiguity, connectivity, number of particle-particle contacts per particle and the particle-particle contact sizes were measured from the simulated microstructures. In the first 2000 time steps of the simulation, particle growth is mainly through precipitation from the liquid since the simulated systems were for convenience initialized with a supersaturated liquid. Fig. 4 shows that the solid volume fraction increases quickly until the liquid has reached its equilibrium composition. From that point, the main driving force for further microstructure evolution is the possibility to reduce the total interface energy in the system, which gives a realistic representation of the grain evolution in the final stage of LPS. The solid volume fraction then remains nearly constant in time and the solid particles grow at a relatively slower rate. In the further discussion, microstructure characteristics are presented as a function of these final solid volume fractions obtained when the liquid is equilibrated. As discussed further, the structures evolve in an almost self-similar way between $t=10^{5}$ and $t=2 \times 10^{5} \triangle t$.

2-D sections of the simulated microstructures at $t=75 \times 10^{3} \triangle t$ are shown in Fig. 5 for different interface energy ratios $\left(\sigma_{s s} / \sigma_{s l}\right)$ and solid phase volume fractions $f_{p}$. We observe again that full wetting of the grains is achieved 
when $\sigma_{s s}=2.5 \sigma_{s l}$. Fig. $5(\mathrm{a}, \mathrm{b}, \mathrm{c})$ show that for $\sigma_{s s}=2.5 \sigma_{s l}$ a network of liquid is maintained in the whole system for all considered solid volume fractions, although the thickness of the liquid film decreases with increasing solid volume fraction. For $\sigma_{s s}=2 \sigma_{s l}$, there is still a large extend of wetting, but there are also a number of particle-particle contacts present, of which the number increases with increasing volume fraction of the solid particles (see Fig. 5(f)). Furthermore, images Fig. 5(g) to Fig. 5(l) show that the particleparticle contacts become more pronounced for higher solid volume fractions and decreasing $\sigma_{s s} / \sigma_{s l}$ ratio. For $\sigma_{s s} / \sigma_{s l}=1$, an almost completely connected grain structure with liquid pockets at the triple junctions is obtained, as shown in Figs. 5(j) to (l).

For $\sigma_{s s}=2 \sigma_{s l}$, it is probable that the number of particle-particle contacts observed in the simulated systems is somewhat exaggerated, since fine liquid boundary layers may not be resolved due to the finite diffuse interface width. However, the general trends observed in the simulations are well in agreement with standard wetting theories. The results presented further in this paper also show that the simulation results reproduce well the important characteristics, experimental data and analytical theories for liquid phase sintering.

As all simulations contain more than 60 vol. $\%$ solid fraction, the initially spherical particles develop flattened faces and go through the shape accommodation process. According to German et al., there are three main mechanisms for particle shape accommodation and contact growth, namely contact flattening, Ostwald ripening of the solid particles and solid state bonding [3]. In contact flattening, capillary stress leads to dissolution of solid in the grain-grain contact areas and preferential precipitation at a location away from the contact. Ostwald ripening involves the dissolution of small particles and coarsening of the larger ones and is the only shape accommodation mechanism that results in an overall increase of the average particle size. Solid state bonding involves the growth of the contact between particles by diffusion of material towards the contact area [53]. The simulated microstructure in Fig. 6 visualizes that the three main mechanisms are reproduced automatically and can be considered simultaneously by the proposed phase field model. The proposed model is thus a powerful tool to analyse microstructure characteristics during liquid phase sintering as a function of system and material properties. 


\subsection{Particle growth and particle size distribution}

Once the liquid has obtained its equilibrium composition, the dominating coarsening mechanism of the solid particles depends on the ratio of the solid-solid and solid-liquid interface energies $\sigma_{s s} / \sigma_{s l}$. For $\sigma_{s s} \geq 2 \sigma_{s l}$, almost full wetting is achieved and the total surface energy can be reduced by Ostwald ripening of the solid particles. This process is controlled by long-range diffusion in the liquid. For $\sigma_{s s}<2 \sigma_{s l}$, the total amount of surface energy can be decreased via grain growth and Ostwald ripening of the liquid pockets. The controlling factor will depend on the relative velocity with which each of these processes can take place, hence on the material parameters (grain boundary mobility and solid diffusion coefficient) and microstructure topology (the fraction of solid-solid and solid-liquid boundaries). For both Ostwald ripening and grain growth, the average particle radius as a function of time is expected to follow a power law,

$$
\left\langle r_{p}(t)\right\rangle^{n}-\left\langle r_{p}(0)\right\rangle^{n}=K t,
$$

with $K$ the rate constant, $\left\langle r_{p}(0)\right\rangle$ the average solid particle or grain radius at $t=0$ and $n$ the growth exponent [54]. In the case of diffusion controlled growth, an exponent $n=3$ is expected. This can be diffusion, through either the liquid or the solid phase. When particle growth is purely controlled by the movement of grain boundaries (grain growth), an exponent $n=2$ is expected.

Fig. 7 shows the average particle size as a function of time for a number of $\sigma_{s s} / \sigma_{s l}$ ratios and $f_{p}$-values considered in the simulations. The growth rate exponents obtained by fitting the power growth law through the data from $t=10^{5}$ till $t=2 \times 10^{5} \triangle t$ are summarized in Table 1 . The growth exponent can be considered as constant over this time interval. We found that in our simulations, the growth exponent $n$ decreases from 3.03 to 2.4 with decreasing $\sigma_{s s} / \sigma_{s l}$ ratio and increasing volume fraction of the solid phase particles $f_{p}$. This dependence of the growth rate exponent on the interface energy ratio can be understood based on the microstructures shown in Fig. 5. Namely, for high $\sigma_{s s} / \sigma_{s l}$ ratio, the microstructures show full wetting. The growth of the particles is through Ostwald ripening with a growth exponent equal to 3 , which is controlled by long-range diffusion through the liquid phase. For decreasing $\sigma_{s s} / \sigma_{s l}$ ratios, the number of flattened particle-particle contacts increases. The amount of coarsening by dissolution-precipitation mediated by the liquid phase is thus reduced $[54,55]$ and the effect of grain growth within clusters of grains gains importance, resulting in a lower growth exponent. 
An exponent $n=3$ is in agreement with previous simulation results for the coarsening of solid particles in a liquid matrix. The values $n<3$ are different from those found in previous simulation studies, where even for non-wetted systems and high volume fractions of the solid phase a growth rate exponent $n=3$ was found $[38,17,18]$. In these previous studies, grain boundary mobility was chosen of the same order or larger than the mobility of the solid-liquid interfaces. Coarsening within clusters of solid particles was therefore found to be fast compared to the overall microstructure evolution, and thus not rate controlling. In our study, the grain boundary mobility is taken approximately 100 times smaller than the mobility of the solid-liquid interface. Coarsening within clusters of solid phase particles then occurs on the same time scale as the coarsening of the solid-phase clusters and liquid pockets. The simulated systems are thus in a regime with mixed control limited by both grain boundary migration and bulk diffusion through the solid phase. Since, growth in the bulk diffusion controlled regime scales with $R^{3}$ and growth in the grain growth controlled regime only with $R^{2}$, the system kinetics will evolve towards a regime with growth controlled by bulk diffusion and a growth exponent $n=3$. The conditions of the considered systems are, however, of such a nature that the transition towards the diffusion controlled regime is extremely slow. The growth rate coefficient, grain size distribution and topological characteristics all appeared constant over the considered simulation time (during which the amount of particles reduced from almost 5000 particles to approximately 300 particles). Growth rate exponents between 2 and 3 are also seen experimentally for high volume fractions [13]. Although the systems with $n<3$ are strictly speaking not in a self-similar regime, the analysis of the simulated microstructures are still of interest to assist the interpretation of experimental findings.

Fig. 8 shows the normalized grain size distributions as obtained from a number of simulations at time steps between $10^{5}$ and $2 \times 10^{5} \triangle t$. This analysis shows clearly that the normalized particle size distributions from simulations with different interface energy ratios and solid volume fractions are very similar. They all fit a Weibull distribution function with shape factor 4.40. This confirms the theory of Fang et al. [56] which states that for LPS, the normalized grain size distribution always evolves towards a same form which is self-similar in time and independent of the initial particle size distribution. An experimental data set obtained for a 78 wt\% tungsten alloy with a $7: 3$ ratio nickel iron binder liquid-phase sintered under microgravity conditions for 2 hours is added to Fig. 8[57], for comparison. The experimental data are 
best described with a Weibull distribution with shape factor 4.22, which is in very good agreement with the distributions obtained from our simulations.

\subsection{Particle-particle contacts and mean apparent dihedral angle}

In the context of liquid phase sintering, contiguity is usually defined as

$$
C_{s s}=S_{s s} /\left(S_{s s}+S_{s l}\right)
$$

in which $S_{s s}$ is the total particle-particle contact area, and $S_{s l}$ the total solid-liquid interface area. It has been shown by German that the contiguity typically stabilizes at a value determined by the surface energy, the solid volume fraction and the grain shape [30].

To calculate the contiguity from equation (14), the total particle-particle contact area and total solid-liquid interface area were obtained from the discrete voxel based representation of the simulated microstructures, as described in section 2.1. The contiguity as obtained in the phase-field simulations as a function of time is plotted in Fig. 9. In all cases, there is a large increase in contiguity at the very beginning of the simulations, when particle growth is dominated by precipitation from the supersaturated liquid and the amount of solid phase is increasing vastly. After this initial period, however, the contiguity is nearly constant over the considered simulation time. As one can expect from the microstructure images in Fig. 5, the contiguity evolves to 0 for $\sigma_{s s} / \sigma_{s l}=2.5$ and a particle volume fraction $f_{p}$ equal to 0.65 and 0.7 , as all grains are completely wetted by the liquid phase. For $\sigma_{s s} / \sigma_{s l}=2.5$ and larger particle volume fractions and for $\sigma_{s s} / \sigma_{s l}=2$ a small, but finite contiguity is measured, although theoretically full wetting is expected. The observation of a finite contiguity for these cases may be attributed to the diffuse character of the interfaces, i.e. the liquid layer which might be present in between these grains is too narrow to be resolved in the simulations. However, also for experiments, the resolution of a micrograph is limited and a finite contiguity may be found for these cases. In general, the contiguity increases with particle volume fraction for a given $\sigma_{s s} / \sigma_{s l}$ ratio and decreases with increasing $\sigma_{s s} / \sigma_{s l}$-ratio for a given particle volume fraction, in agreement with general experimental observations [3].

In Table 1, the contiguity is listed together with the growth exponent for different simulated systems, indicating a close correlation between contiguity and growth rate exponent. For a contiguity close to 0 , growth is controlled by long range diffusion through the liquid phase and a growth rate exponent 
close to 3 is obtained. For increasing contiguity, corresponding to an increasing number of particle-particle contacts, the growth rate exponent decreases. It would be highly interesting to study the correlation between contiguity and growth rate exponent and the effects of grain boundary mobility, diffusivities and time on this, in more detail. However, as it appeared that a comprehensive parameter study, considering a wide range of ratios of the grain boundary mobility with respect to the diffusivities of the phases, is required to reveal insights, this was considered as outside the scope of this paper.

Fig. 10 presents the distribution of the number of particle-particle contacts per particle $N_{c}$ for $\sigma_{s s}=1.7 \sigma_{s l}$ and $f_{p}=0.65, f_{p}=0.70$ and $f_{p}=0.78$ as obtained from the voxel based representation of the 3-D simulation images. It is evident that the distribution is strongly influenced by the solid volume fraction. A higher solid content resulted in a broader distribution of the number of contacts per particle. Experimental data obtained by Rowenhorst [13] for systems containing Sn-rich particles dispersed in a Pb-Sn eutectic matrix are added to Fig. 10 for comparison. There is a very good agreement between the simulation and experimental data for the same solid volume fraction, as can be seen for $f_{p}=0.78$. Moreover, the experimental data show the same particle volume fraction dependence as the data obtained from the phase field simulations. Fig. 11, shows the accumulated particle-particle contact size distributions obtained for different $\sigma_{s s} / \sigma_{s l}$ ratios and at different times. The different curves fall together. The solid line represents a Weibull distribution with shape factor 2.5 which matches the accumulated distributions obtained from the simulations well.

To evaluate the accuracy with which dihedral angles are reproduced in the simulations, simulations were performed of the evolution of two spherical solid particles of equal size in a liquid phase matrix. Fig. 12 presents images at different time steps of such a phase-field simulation for the cases $\sigma_{s s}=1.7 \sigma_{s l}$ and $\sigma_{s s}=\sigma_{s l}$, for which equilibrium dihedral angles $2 \sigma_{s l} \cos \phi^{*} / 2=\sigma_{s s}$, $\phi^{*}=63.57^{\circ}$ and $\phi^{*}=120^{\circ}$ are expected. The apparent dihedral angle in the simulated images is measured as a function of time by fitting a circle through the contours $H_{s}=H_{l}=0.5$ (with $H_{s}$ and $H_{l}$ as defined in Equations (4)), as explained in [36]. Fig. 12(a) to Fig. 12(d) and Fig. 12(e) to Fig. 12(h) reveal that with the proposed model and parameter relations the equilibrium dihedral angles are reproduced with high accuracy in the simulations (Figs. 12(d) and 12(h)), as was also shown in [43, 44]; however, also that it takes time before the equilibrium dihedral angles are reached 
at particle-particle contacts, as diffusional transport over longer distances is needed. This is different from grain growth in single-phase materials where it can often be assumed that the equilibrium dihedral angles are obtained instantaneously. In LPS, the apparent dihedral angle between solid-liquid interfaces, measured at a certain moment, can thus be smaller than the equilibrium dihedral angle. As shown in figures 12, it changes with increasing contact size. The equilibrium dihedral angle is reached when the maximum neck size for the involved particle sizes is reached. This was also observed and studied experimentally [3, 27, 28, 29].

We were not able to measure the apparent dihedral angles from the $3-\mathrm{D}$ microstructures accurate enough for a reliable analysis. However, we will apply empirical relations between the mean apparent dihedral angle, on the one hand, and contiguity and connectivity, on the other hand.

The following expression from German [55] relates the contiguity $C_{s s}$, solid volume fraction $f_{p}$ and the mean apparent dihedral angle $\phi$ :

$$
C_{s s}=f_{p}^{2}\left[0.43 \sin (\phi)+0.35 \sin ^{2}(\phi)\right] .
$$

Fig. 13 presents the contiguity as a function of solid volume fraction as obtained from the simulated systems. Higher $f_{p}$ and lower $\sigma_{s s} / \sigma_{s l}$ result in a higher contiguity. Fitting of equation (15) through these data points, results in values for the mean apparent dihedral angle at particle-particle contacts equal to $\phi=3^{\circ}$ for $\sigma_{s s} / \sigma_{s l}=2.5, \phi=23^{\circ}$ for $\sigma_{s s} / \sigma_{s l}=2, \phi=50^{\circ}$ for $\sigma_{s s} / \sigma_{s l}=1.7$ and $\phi=90^{\circ}$ for $\sigma_{s s} / \sigma_{s l}=1$. For the cases $\sigma_{s s} / \sigma_{s l}=2.5$ and $\sigma_{s s} / \sigma_{s l}=2$ (full wetting), an apparent dihedral angle $\phi=0^{\circ}$ is expected. The fact that we obtain a finite value for the apparent dihedral angle is related to the small, but finite, contiguity values obtained for these systems. For the other cases, $\sigma_{s s} / \sigma_{s l}<2$ (no wetting), the mean apparent dihedral angle is smaller than the equilibrium angle.

Experimentally, it was also found that the connectivity and the 3-D coordination number $N_{c}$ are related to the mean apparent dihedral angle $\phi$ as [58]

$$
C_{g}=K N_{c} \sin \left(\frac{\phi}{2}\right) .
$$

Connectivity is defined as the mean number of particle-particle contacts of the particles as measured from a 2-D section of the microstructure. The 3 -D coordination number can be obtained as the average number of particleparticle contacts of the particles measured from the $3-\mathrm{D}$ microstructure. It 
was determined by Yang and German that for liquid phase sintering, the value of $K$ is 0.68 [59]. Equation (16) can thus be used to estimate the mean 3-D apparent dihedral angle in the simulations based on the measured connectivities and 3-D coordination numbers.

Table 2 lists the mean apparent dihedral angels $\phi_{2}$ predicted using Eq. (16), together with the measured properties needed for the calculations. Since the number of particle-particle contacts for $\sigma_{s s} / \sigma_{s l}=2.5$ and 2 is low, we only considered the systems with $\sigma_{s s} / \sigma_{s l}$ equal to 1.7 and 1 . The mean apparent dihedral angles $\phi_{1}$ obtained from the contiguity measurements using Eq. (15) are added for comparison.

Both estimates agree well and it is possible that the mean apparent dihedral angle deviates from the equilibrium angle. However, relations (15) and (16) were derived based on several assumptions, which may be inaccurate for the considered particle size distribution. The contiguity relation (Equation (15)) assumes a mono-sized particle distribution, while in the simulated microstructures, the particle sizes are distributed and contacting particles are of a different size. German et al. (see Fig.29 in [3]) namely showed that for a given contiguity, interfacial energies and solid volume fraction, the apparent dihedral angle is larger in a system with distributed particle sizes than for mono-sized particles. For the connectivity relation (Equation (16)), the choice of the value of the $\mathrm{K}$ parameter is critical. It depends on the grain size distribution and solid volume fraction and the estimation of Yang and German [59] of $K=0.68$ may not apply to the microstructures considered in our simulations. Liu et al. [60] showed that the value of $\mathrm{K}$ may be smaller for a system with distributed particle sizes and for lower solid volume fractions.

\section{Conclusion}

A novel 3-dimensonal phase-field model designed to study grain structure evolution in the final stage of liquid phase sintering is presented and implemented using a bounding box sparse data structure algorithm. The model considers the evolution of binary polycrystalline solid-liquid systems and allows to vary the solid-solid grain boundary energies and solid-liquid interface energies independently, as well as the equilibrium compositions and diffusion properties of the liquid and the solid. Simulations were performed for $\sigma_{s s} / \sigma_{s l}$ ratios varying between 1 and 2.5 and $f_{p}$ between 0.65 and 0.83 , starting from systems containing initially 5000 solid particles randomly placed without overlap. 
For $\sigma_{s s}>2 \sigma_{s l}$, there is complete wetting of all grains by the liquid phase. For lower $\sigma_{s s} / \sigma_{s l}$-ratios, the amount of particle-particle contact increases with decreasing $\sigma_{s s} / \sigma_{s l}$ and increasing $f_{p}$. For $\sigma_{s s}=\sigma_{s l}$ and the considered particle volume fractions, all grains are in contact and the liquid phase is distributed as liquid pockets at the grain boundaries.

In the considered simulations, the exponent $n$ in the power growth law varies from 2.4 to 3 , indicating a transition in the coarsening mechanism of the particles from a coarsening regime with mixed control limited by bulk diffusion through the solid and/or liquid and grain boundary migration $n<3$ to coarsening controlled by bulk diffusion through the liquid phase $(n=3)$, i.e. Ostwald ripening. The measured growth exponents correlate with the measured contiguities and connectivities. Strictly speaking, the structures with $n<3$ are not yet in a self-similar regime and will eventually evolve towards a self-similar regime controlled by bulk diffusion; however, for the considered simulations the transition towards this self-similar regime is so slow that the growth rate exponent and other microstructure characteristics appeared self-similar over an extended simulation time.

The average apparent dihedral angle was estimated for different $\sigma_{s s} / \sigma_{s l^{-}}$ ratios based on empirical relations from literature. The values obtained based on contiguity measurements agreed well with those obtained based on connectivity measurements. They deviated however considerably from the theoretical dihedral angles expected based on Young's equations. This deviation can be explained by the long range diffusion required to adapt the dihedral angles between solid-liquid interfaces and by taking the effects of the particle size distribution into account.

The simulation data are in good agreement with available experimental data from several material systems.

The presented results show that the proposed model describes automatically the main mechanisms taking place in the final stage of liquid phase sintering, and that dihedral angles are reproduced with high accuracy. The presented model can be further used to study the relationship between contiguity (and other topological characteristics) and the growth rate exponent in more detail and for a wide range of grain boundary mobilities, liquid and solid phase diffusivities, particle volume fractions and $\sigma_{s s} / \sigma_{s l}$-ratios. Such insights are important to interpret experimental observations and would even allow us to link experimentally measured growth rates and/or microstructure characteristics to material properties. Furthermore, it is straightforward to extend the presented approach to simulate microstructure evolution during 
coarsening processes in 3-D polycrystalline two- and three-phase systems $[62,63]$.

\section{Acknowledgments}

This work was supported by the 'Strategic Initiative Materials' in Flanders (SIM) and the Institute for Innovation through Science and Technology in Flanders (IWT) under the Solution based Processing of Photovoltaic Modules (SoPPoM) program. The simulations were performed using the VSC Flemish Supercomputing Center (Vlaams Supercomputer Centrum) funded by the Hercules foundation and the Flemish Government - Department EWI. Dr. Eckard Specht is acknowledged for providing us with the particle packing algorithm.

\section{References}

[1] Shirazi SFS, Gharehkhani S, Mehrali M, et al. (2015) A review on powderbased additive manufacturing for tissue-engineering: selective laser sintering and inkjet 3D printing: Sci. Technol. Adv. Mater. 16: 033502 (20pp)

[2] German RM and Olevsky EA (1998) Modeling grain growth dependence on the liquid content in liquid-phase sintered materials, Metall. Trans. A 29: $3057-3067$

[3] German RM, Suri P and Park SJ (2009) Review: liquid phase sintering, J. Mater. Sci 44: 1-39

[4] Greenwood GW (1956) The growth of dispersed precipitates in solutions, Acta Metall. 4: 243-248

[5] Lifshitz IM and Slyozov VV (1961) The Kinetics of Precipitation from Supersaturated Solid Solutions, J. Phys. Chem. Solids 19: 35-50

[6] Wagner C (1961) Theory of the aging of precipitates by dissolutionreprecipitation (Ostwald ripening), Electrochem 65: 581-591

[7] Ardell AJ (1972) The effect of volume fraction on particle coarsening: theoretical considerations, Acta Metall. 20: 61-71 
[8] Tsumuraya K and Miyata Y (1983) Coarsening models incorporating both diffusion geometry and volume fraction of particles, Acta Metall. 31: $437-452$

[9] Voorhees PW and Glicksman ME (1984) Solution to the multiparticle diffusion problem with applications to ostwald ripening, Acta Metall. 32: 2001-2011

[10] Yao JH, Elder KR, Guo H and Grant M (1993) Theory and simulation of Ostwald ripening, Phys. Rev. B 47: 14110-14125

[11] Dehoff RT (1991) A geometrically general theory of diffusion controlled coarsening, Acta metall. 39: 2349-2360

[12] Fan D, Chen SP, Chen LQ and Voorhees PW (2002) Phase-field simulation of 2-D Ostwald ripening in the high volume fraction regime, Acta Mater. 50: 1895-1907

[13] Rowenhorst DJ, Kuang JP, Thornton K and Voorhees PW (2006) Threedimensional analysis of particle coarsening in high volume fraction solidliquid mixture, Acta Mater. 54: 2027-2039

[14] Kim SG (2007) Large-scale three-dimensional simulation of Ostwald ripening, Acta Mater. 55: 6513-6525

[15] Wang KG, Ding X, Chang K and Chen LQ (2010) Phase-field simulation of phase coarsening at ultrahigh volume fractions, J. Appl. Phys. 107: 061801-061808

[16] Streitenberger P (2013) Analytical description of phase coarsening at high volume fractions, Acta Mater. 61: 5026-5035

[17] Lee SB, Rickman JM, and Rollett AD (2007) Three-dimensional simulation of isotropic coarsening in liquid phase sintering: A model, Acta Mat. 55:615-626

[18] Spett A, Wimmer R, Werz T, Heinze M, Odenbach S, Krill CE III, and Schmidt V (2015) Stochastic 3D modeling of Ostwald ripening at ultrahigh volume fractions of the coarsening phase, Modelling Simul. Mater. Sci. Eng. 23:065001 
[19] Wang YU (2006) Computer modeling and simulation of solid-state sintering: A phase-field approach: Acta Mater. 54: 953-961

[20] Asp K, Agren J (2006) Phase-field simulation of sintering and related phenomena - A vacancy diffusion approach: Acta Mater. 54: 1241-1248

[21] Bjork R, Tikare V, Frandsen HL, Pryds N (2013) Effect of particle size distributions on the microstructural evolution during sintering: J. Am. Ceram. Soc. 96: 103-110

[22] Shinagawa K (2014) Simulation of grain growth and sintering process by combined phase-field/discrete-element method: Acta Mater. 66: 360-369

[23] Shinagawa K, Maki S, Yokota K (2014) Phase-field simulation of platelike grain growth during sintering of alumina: J. Europ. Ceram. Soc. 34: 3027-3036

[24] Tikare V, Cawley JD (1998) Numerical simulation of grain growth in liquid phase sintered materials - II Study of isotropic grain growth: Acta Mater. 46: 1343-1356

[25] Tikare V, Cawley JD (1998) Numerical simulation of grain growth in liquid phase sintered materials - I Model: Acta Mater. 46: 1333-1342

[26] Svoboda J, Riedel H, Gaebel R (1996) A model for liquid phase sintering: Acta Mater. 44: 3215-3226

[27] Riegger OK and Vlanck LH (1960) Dihedral angle measurement, Metall. Soc.AIME, Trans 218: 933-935

[28] Chillar P, Sangal S and Upadhyaya A (2004) Automated dihedral angle measurement in liquid phase sintered alloys, Int. J. Mater. Res. 95: 3-7

[29] Ye S, He Y and Smith JE (2003) Dihedral angle measurement in microgravity liquid phase sintered microstructures, J. Mater. Sci. 38: 377-388

[30] German RM (1985) Liquid Phase Sintering, Metall. Trans. A 16: $1247-$ 1252

[31] Johnson JL, Upadhyaya A and German RM (1998) Microstructural effects on distortion and solid-liquid segregation during liquid phase sintering under microgravity conditions, Metall. Trans. B 29: 857-866 
[32] Liu Jianxin and German RM (2000) Microstructural parameters related to liquid-phase sintering, Metall. Trans. A 31: 2607-2614

[33] Moelans N, Blanpain B and Wollants P (2008) Quantitative analysis of grain boundary properties in a generalized phase field model for grain growth in anisotropic systems, Phys. Rev. B 78: 024113-024123

[34] Moelans N, Blanpain B and Wollants P (2008) Quantitative phase-field approach for simulating grain growth in anisotropic systems with arbitrary inclination and misorientation dependence, Phys. Rev. Lett. 101: 025502025502

[35] Folch R and Plapp M (2005) Quantitative phase-field modeling of twophase growth, Phys. Rev. E 72: 011602-011627

[36] Moelans N, Wendler F and Nestler B (2009) Comparative study of two phase-field models for grain growth, Comput. Mater. Sci. 46: 479-490

[37] Garcke H, Nestler B and Stoth B (1999) SIAM J. Appl. Math. 60: 295

[38] Poulsen S, Voorhees P and Lauridsen E (2013) Acta Mater. 61: 12201228

[39] Fan D and Chen LQ (1997) Acta Mater. 45:4145-4154

[40] Kazaryan A, Wang Y, Dregia SA and Patton BR (2002) Grain growth in anisotropic systems: comparison of effects of energy and mobility, Acta Mater. 50: 2491-2502

[41] Moelans N (2011) A quantitative and thermodynamically consistent phase-field interpolation function for multi-phase systems, Acta Mater. 59: $1077-1086$

[42] Chen LQ, Yang W (1994) Computer simulation of the domain dynamics of a quenched system with a large number of non conserved order parameters: The grain-growth kinetics, Phys Rev B50:15752

[43] Tang M, Reed BW and Kumar M (2012) Coarsening kinetics of topologically highly correlated grain boundary networks, J. Appl. Phys 112: 043505-043514 
[44] Chang K and Moelans N (2014) Effect of grain boundary energy anisotropy on highly textured grain structures studied by phase-field simulations, Acta Mater. 64: 443-454

[45] Moelans N, http://nele.studentenweb.org/docs/GammaDependence.txt

[46] Andersson JO and Ågren J (1992) Models of numerical treatment of multicomponent diffusion in simple phases, J. Appl. Phys 72: 1350-1355

[47] Kim SG (2007) A phase-field model with antitrapping current for multicomponent alloys with arbitrary thermodynamic properties, Acta Mater. 55: 4391-4399

[48] Vanherpe L, Moelans N, Blanpain B and Vandewalle S (2011) Bounding box framework for efficicent phase-field simulation of grain growth in anisotropic systems, Comput. Mater. Sci. 50: 2221-2231

[49] Vanherpe L, Moelans N, Blanpain B and Vandewalle S (2007) Bounding box algorithm for three-dimensional phase field simulations of microstructural evolution in polycrystalline materials, Phys. Rev. E 76: 056702056702

[50] Aarts E and Lenstra JK (1997) Local Search in Combinatorial Optimization, John Wiley Sons, New York

[51] Specht E (2014), www.packomania.com

[52] Ravash H, Specht E, Vleugels J, Moelans N (2014) 3D phase-field simulation and characterization of microstructure evolution during Liquid Phase Sintering, Advances in Science and Technology (Proceedings of CIMTEC, 13th International Ceramics Congress) 87:132-138

[53] Gessinger GH, Fischmeister HF and Lukas HL (1973) A model for second-stage liquid-phase sintering with a partially wetting liquid, Acta Metall. 21: $715-724$

[54] German RM (1985) Liquid phase sintering, Plenum press, New York

[55] German RM (1996) Sintering Theory and Practice, Wiley, New York

[56] Fang Z, Patterson BR and Turner ME (1992) Influence of particle size distribution on coarsening, Acta Metall Mater. 40: 713-722 
[57] Liu Y, German RM and Iacocca RG (1999) microstructure quantification procedures in liquid-phase sintered materials, Acta mater. 47: 915-926

[58] German RM (1987) The two-dimensional connectivity of liquid phase sintered microstructures, Metall. Trans. A 18: 909-914

[59] Yang SC, German RM and Iacocca RG (1991) Gravitational limit of particle volume fraction in liquid-phase sintering, Metall. Trans. A 22: 786-791

[60] Liu PL and Lin SH (2002) The K value distribution of liquid phase sintered microstructure, MATER T JIM 43: 2115-2119

[61] Johnson KL, Campbell LG, Park SJ and German RM (2009) Grain Growth in Dilute Tungsten Heavy Alloys during Liquid-Phase Sintering under Microgravity Conditions, Metall. Trans. A 40: 426-427

[62] Ravash H, Vleugels J, Moelans N (2014) Three-dimensional phase-field simulation of microstructural evolution in three-phase materials with different diffusivities, J. of Materials Science (JMSC) 49: 7066-7072

[63] Yadav V, Vanherpe L, Moelans N (2016) Effect of volume fractions on microstructure evolution in isotropic volume-conserved two-phase alloys: A phase-field study, Comp. Mater. Sci. 125: 297-308 


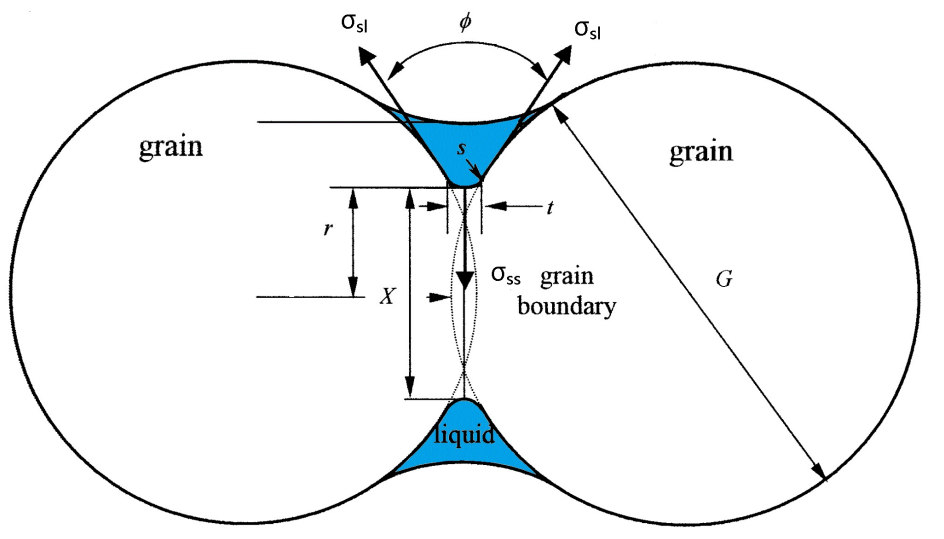

Figure 1: Schematic illustration of the neck and apparent dihedral angle $\phi$ formed at the contact between two solid particles in a surrounding liquid phase. $r, s, t$ and $X$ are the neck radius, neck surface radius of curvature, neck thickness and contact size, respectively $[3]$. 

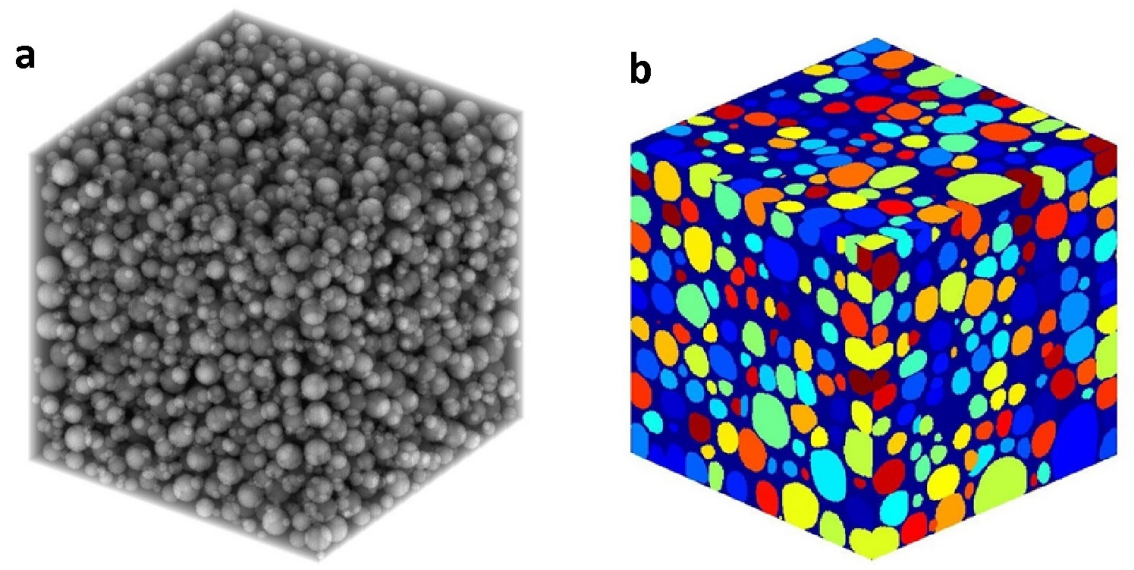

Figure 2: (a) Example of an initial structure used in the 3-D simulations with 5000 particles distributed over the system for $f_{p}=0.67$. (b) Discretized voxel representation of the simulated microstructure for $\sigma_{s s} / \sigma_{s l}=2$ and $f_{p}=0.7$ at $t=5 \times 10^{4} \triangle t$.

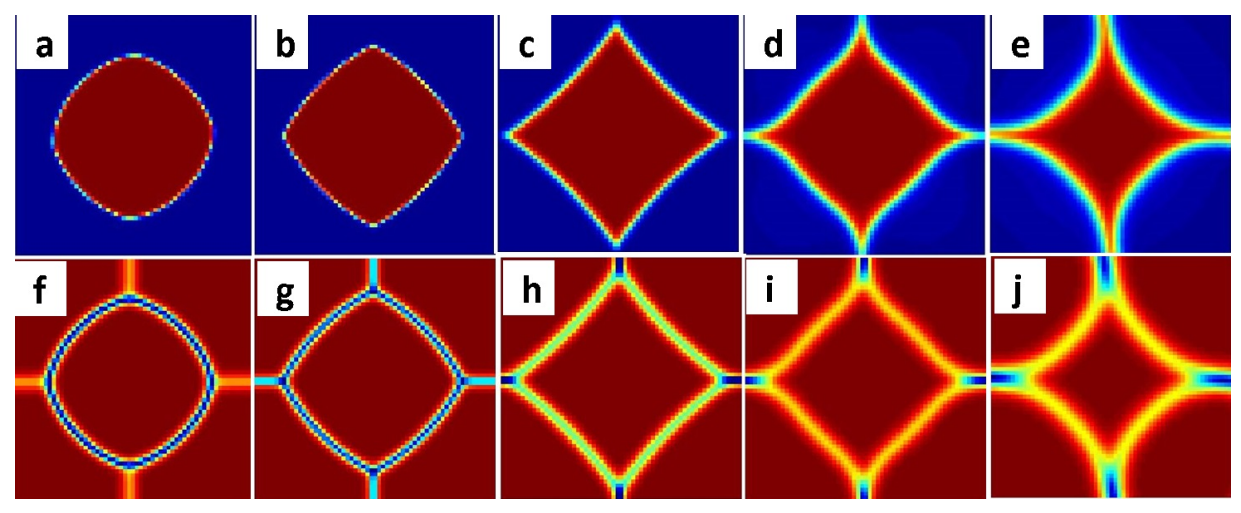

Figure 3: Simulation results obtained for different $\sigma_{s s} / \sigma_{s l}$ ratios considering 4 neighboring grains with a liquid pocket at their junction: $(\mathrm{a}, \mathrm{f}) \sigma_{s s} / \sigma_{s l}=0.5,(\mathrm{~b}, \mathrm{~g}) \sigma_{s s} / \sigma_{s l}=1$, (c, h) $\sigma_{s s} / \sigma_{s l}=1.7,(\mathrm{~d}, \mathrm{i}) \sigma_{s s} / \sigma_{s l}=2$ and $(\mathrm{e}, \mathrm{j}) \sigma_{s s} / \sigma_{s l}=2.5$. The first row represents the composition maps and the second row $\sum_{i=1}^{p_{s}+1} \eta_{i}^{2}$, where the sum is taken over all phase-fields. The shape of the liquid pocket varies with $\sigma_{s s} / \sigma_{s l}$. 


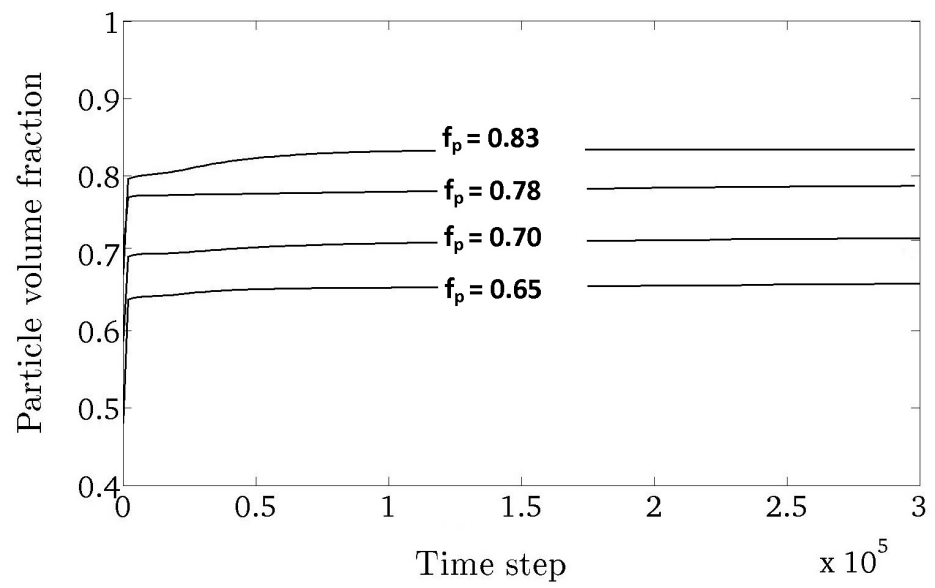

Figure 4: Variation of the particle volume fraction in time for the simulated systems. After approximately 2000 time steps, a particle volume fractions $f_{p}=0.65,0.70,0.78$ and 0.83 are obtained for the initial average particle sizes and initial supersaturated liquid compositions $\left\langle r_{p}\right\rangle=5.94 \triangle x$ and $c_{l}^{0}=0.85,\left\langle r_{p}\right\rangle=6.31 \triangle x$ and $c_{l}^{0}=0.85,\left\langle r_{p}\right\rangle=6.71 \triangle x$ and $c_{l}^{0}=0.85$, and $\left\langle r_{p}\right\rangle=6.71 \triangle x$ and $c_{l}^{0}=0.88$, respectively.

Table 1: The maximum likelihood estimates of the grain growth exponent for selected simulations, as a function of the interface energy ratio and particle volume fraction, together with the measured contiguity $C_{s s}$.

\begin{tabular}{lccc}
\hline Interface energy ratios & $f_{p}$ & $n$ & $C_{s s}$ \\
\hline$\sigma_{s s}=2.5 \sigma_{s l}$ & 0.78 & 3.03 & 0.02 \\
$\sigma_{s s}=2 \sigma_{s l}$ & 0.78 & 2.98 & 0.13 \\
$\sigma_{s s}=1.7 \sigma_{s l}$ & 0.78 & 2.82 & 0.28 \\
$\sigma_{s s}=1 \sigma_{s l}$ & 0.78 & 2.78 & 0.46 \\
$\sigma_{s s}=1 \sigma_{s l}$ & 0.83 & 2.4 & 0.52 \\
\hline
\end{tabular}



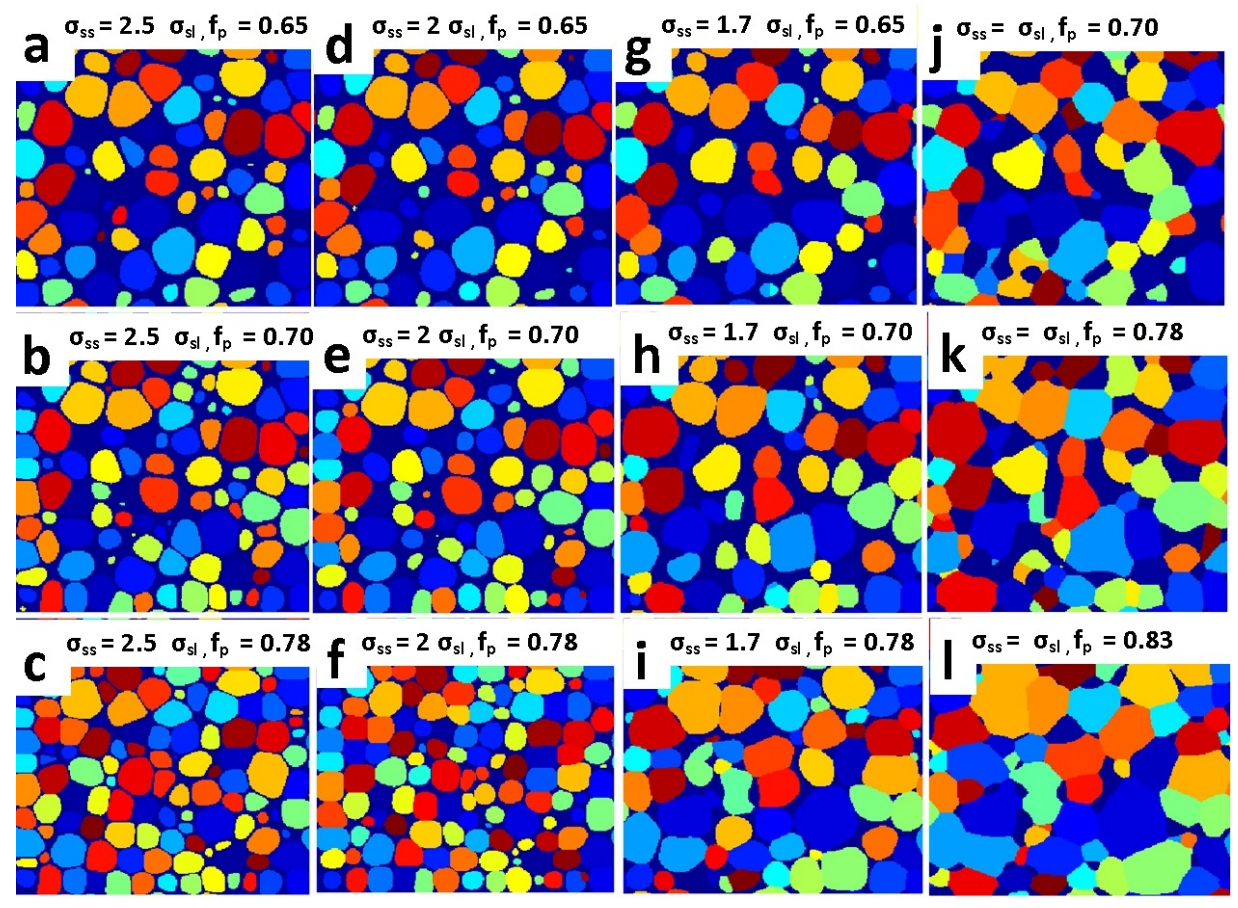

Figure 5: Sections of the 3-dimensional simulated microstructures for different solid volume fractions $f_{p}$ and solid-solid to liquid-solid interfacial energy ratios $\sigma_{s s} / \sigma_{s l}$, all taken at time $t=75 \times 10^{3} \triangle t$.

Table 2: The estimated mean apparent dihedral angles for a selected number of simulations. $\phi_{1}$ is the mean apparent dihedral angle obtained based on the contiguity measurements. $\phi_{2}$ is the mean apparent dihedral angle obtained based on the grain connectivity measurements and 3-D coordination number $N_{c}$.

\begin{tabular}{lccccr}
\hline LPS simulation & $C_{g}$ & $K$ & $N_{c}$ & $\phi_{1}$ & $\phi_{2}$ \\
\hline$\sigma_{s s}=1.7 \sigma_{s l}, f_{p}=0.65$ & 2.05 & 0.68 & 6.7 & $50^{\circ}$ & $55.7^{\circ}$ \\
$\sigma_{s s}=1.7 \sigma_{s l}, f_{p}=0.70$ & 2.22 & 0.68 & 7 & $50^{\circ}$ & $55.5^{\circ}$ \\
$\sigma_{s s}=1.7 \sigma_{s l}, f_{p}=0.78$ & 2.40 & 0.68 & 7.8 & $50^{\circ}$ & $53.8^{\circ}$ \\
$\sigma_{s s}=\sigma_{s l}, f_{p}=0.70$ & 3.38 & 0.68 & 6.6 & $90^{\circ}$ & $97.7^{\circ}$ \\
$\sigma_{s s}=\sigma_{s l}, f_{p}=0.78$ & 3.65 & 0.68 & 7.4 & $90^{\circ}$ & $93^{\circ}$ \\
$\sigma_{s s}=\sigma_{s l}, f_{p}=0.83$ & 3.90 & 0.68 & 8.2 & $90^{\circ}$ & $88.8^{\circ}$ \\
\hline
\end{tabular}




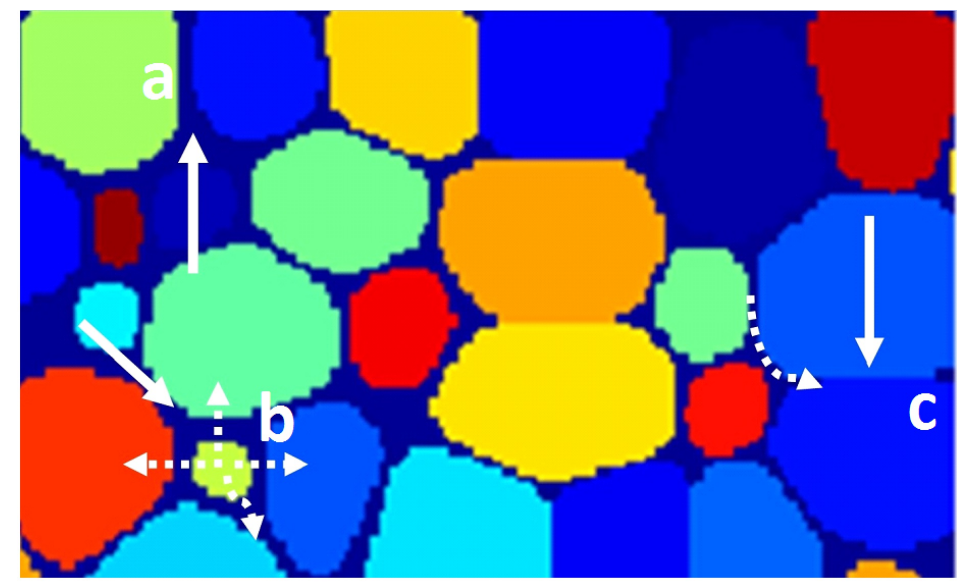

Figure 6: Discretized voxel representation of the simulated microstructure for $\sigma_{s s} / \sigma_{s l}=2$ and $f_{p}=0.78$ at $t=75 \times 10^{3} \Delta \mathrm{t}$. It is illustrated that the three main mechanisms described by German [3] of shape accommodation and contact growth in LPS are reproduced in the simulations: (a) contact flattening, (b) Ostwald ripening and (c) particle-particle contact formation and growth.

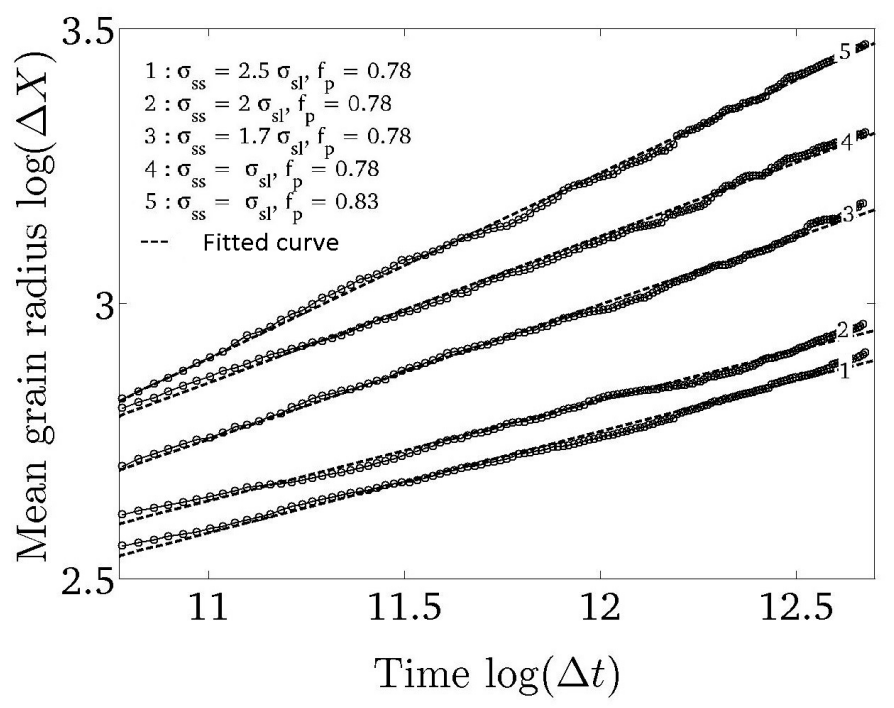

Figure 7: Average particle radius as a function of time plotted for a selected number of the phase-field simulations performed in this study. 


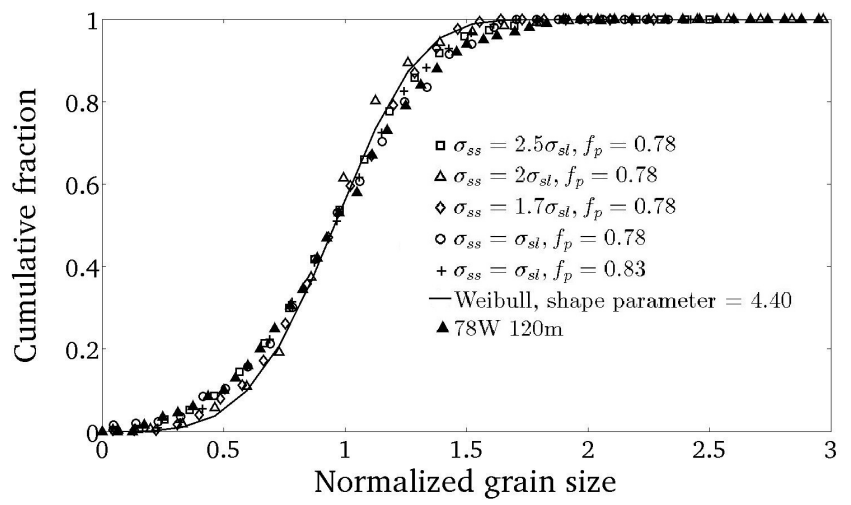

Figure 8: Cumulative distribution of the normalized particle sizes obtained in the simulations after $t=10^{5} \triangle t$, plotted for a selected number of the simulated systems considered in this study. The experimental data obtained for a $78 \mathrm{wt} \% \mathrm{~W}$ alloy $[57,61]$ are added.

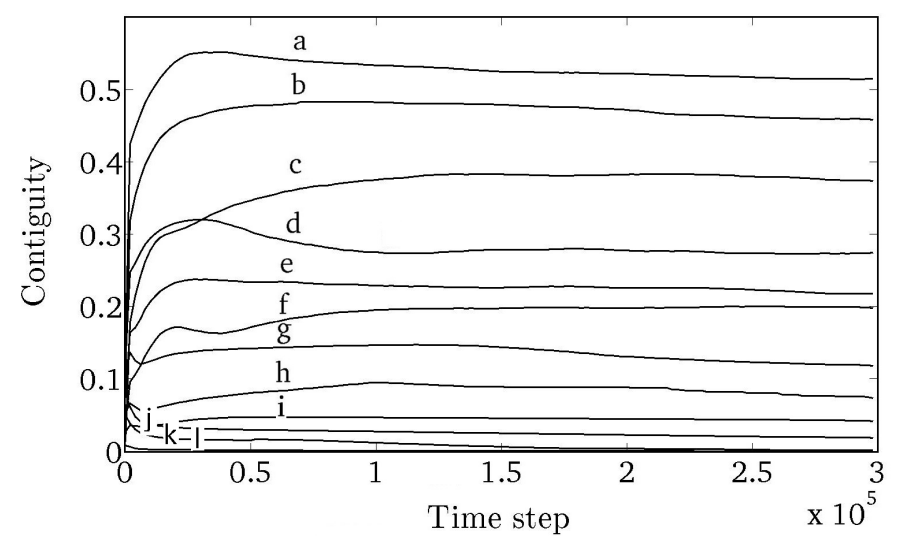

Figure 9: Measured contiguity versus time as obtained for the simulations with (a) $\sigma_{s s}=$ $\sigma_{s l}, f_{p}=0.83$, (b) $\sigma_{s s}=\sigma_{s l}, f_{p}=0.78$, (c) $\sigma_{s s}=\sigma_{s l}, f_{p}=0.70$, (d) $\sigma_{s s}=1.7 \sigma_{s l}$, $f_{p}=0.78$, (e) $\sigma_{s s}=1.7 \sigma_{s l}, f_{p}=0.70$, (f) $\sigma_{s s}=1.7 \sigma_{s l}, f_{p}=0.65$, (g) $\sigma_{s s}=2 \sigma_{s l}$, $f_{p}=0.78$, (h) $\sigma_{s s}=2 \sigma_{s l}, f_{p}=0.70$, (i) $\sigma_{s s}=2 \sigma_{s l}, f_{p}=0.65$, (j) $\sigma_{s s}=2.5 \sigma_{s l}, f_{p}=0.78$, (k) $\sigma_{s s}=2.5 \sigma_{s l}, f_{p}=0.70$, (l) $\sigma_{s s}=2.5 \sigma_{s l}, f_{p}=0.65$. 


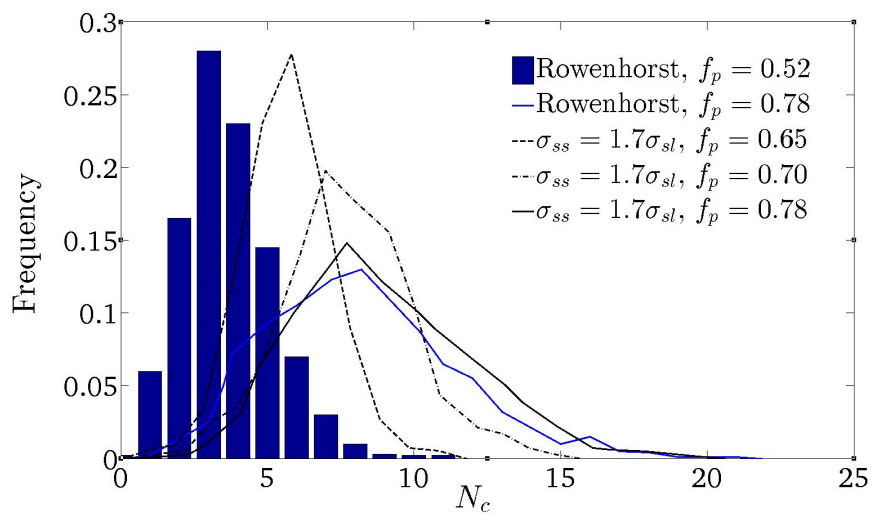

Figure 10: Distribution of the number of contacting particles per particle, $N_{c}$, for $\sigma_{s s}=$ $1.7 \sigma_{s l}$ and $f_{p}=0.65, f_{p}=0.70$ and $f_{p}=0.78$. For comparison, the particle contact distributions from a 3-D structure of Sn-rich particles in a $\mathrm{Pb}-\mathrm{Sn}$ matrix with $f_{p}=0.52$ and $f_{p}=0.78[13]$ are plotted as well.

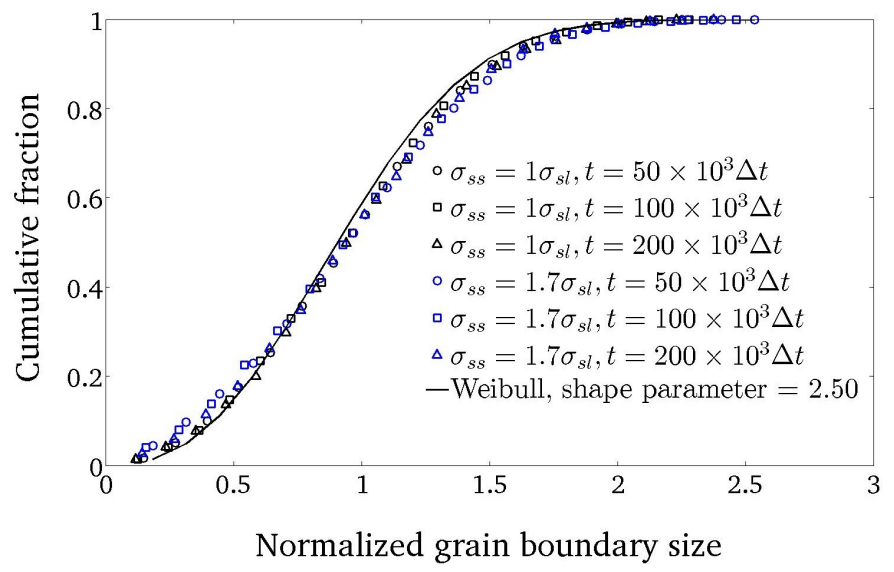

Figure 11: Accumulated particle-particle contact radius distribution for simulations with $\sigma_{s s}=1.7 \sigma_{s l}$ and $\sigma_{s s}=\sigma_{s l}$ at time steps $t=5 \times 10^{4} \Delta t, t=10^{5} \Delta t$ and $t=2 \times 10^{5} \Delta t$. The solid line is a Weibull fit with shape parameter $c=2.5$. 


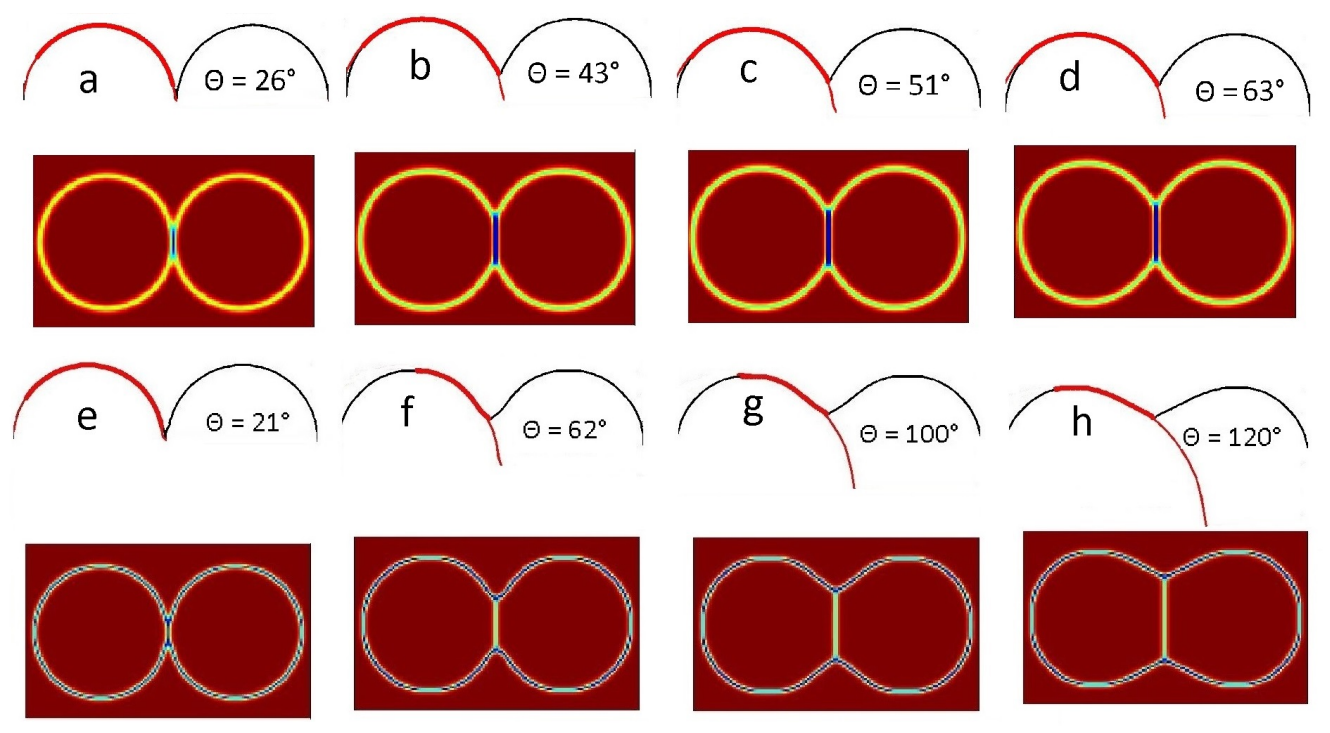

Figure 12: Evolution of the apparent dihedral angle, measured from the simulated images as explained in [36] at (a, e) $t=300 \Delta t$, (b, f) $t=2 \times 10^{4} \Delta t$, (c, g) $t=8 \times 10^{4} \Delta t$ and (d, h) $t=15 \times 10^{4} \Delta t$ for two contacting spheres with initial radius $r=30 \triangle x$ and interfacial energy ratio $\sigma_{s s} / \sigma_{s l}=1.7$ for (a to d) and $\sigma_{s s} / \sigma_{s l}=1$ for (e to h). The apparent dihedral angle increases over time until it reaches the equilibrium value imposed by Young's law.

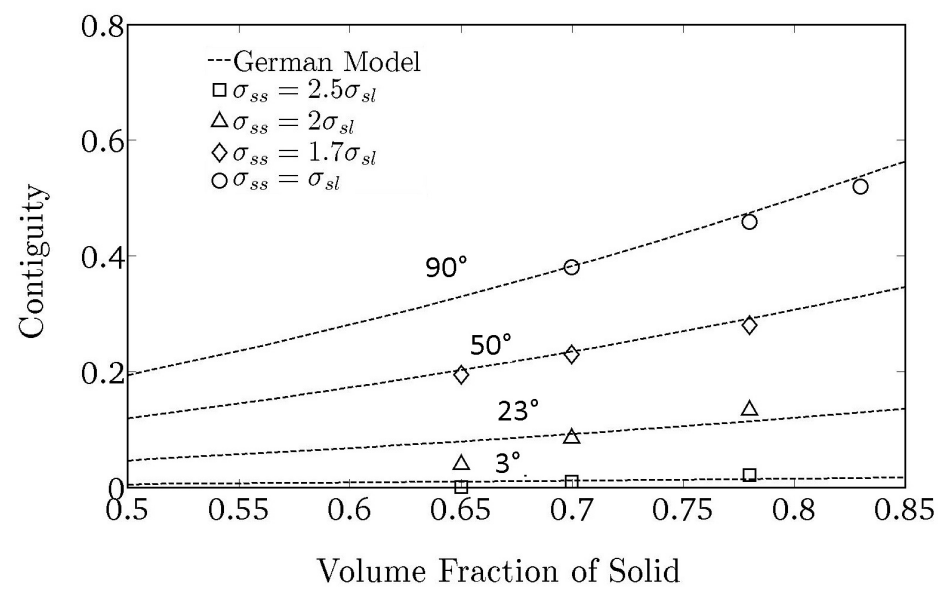

Figure 13: Final contiguity versus solid volume fraction for different $\sigma_{s s} / \sigma_{s l}$-ratios obtained from the simulated microstructures. The dashed lines give the contiguity as a function of particle volume fraction as calculated from Eq. (15) using the indicated mean apparent dihedral angles. 